\title{
ANALYTIC VARIETIES VERSUS INTEGRAL VARIETIES OF LIE ALGEBRAS OF VECTOR FIELDS
}

\author{
HERWIG HAUSER AND GERD MÜLLER
}

\begin{abstract}
We associate to any germ of an analytic variety a Lie algebra of tangent vector fields, the tangent algebra. Conversely, to any Lie algebra of vector fields an analytic germ can be associated, the integral variety. The paper investigates properties of this correspondence: The set of all tangent algebras is characterized in purely Lie algebra theoretic terms. And it is shown that the tangent algebra determines the analytic type of the variety.
\end{abstract}

Local analytic varieties, defined as zero sets of complex analytic functions, can equally be considered as integral varieties associated to certain Lie algebras of vector fields. This is the theme of the present note. As a consequence one obtains a new way of studying singularities of varieties by looking at their Lie algebra. It turns out that the Lie algebra determines completely the variety up to isomorphism. Thus one may replace, to a certain extent, the local ring of functions on the variety by the Lie algebra of vector fields tangent to the variety.

We shall give a brief account of these observations. Details will appear elsewhere, see [HM1, HM2]. The paper of Omori [O], which treats the same topic in a special case, served us as a valuable source of inspiration. Various ideas are already apparent there.

Consider a germ $X$ of a complex analytic variety embedded in some smooth ambient space, $X \subset\left(\mathbb{C}^{n}, 0\right)$. In this note, germ of variety shall always mean reduced but possibly reducible complex space germ. We associate to $X$ the Lie algebra $\mathbb{D}_{X}$ of vector fields on $\left(\mathbb{C}^{n}, 0\right)$ tangent to $X$. To do so let $\mathbb{D}$ denote the Lie algebra of germs of analytic vector fields on $\left(\mathbb{C}^{n}, 0\right)$. We identify $\mathbb{D}$ with Der $\mathscr{O}_{n}$, the Lie algebra of derivations of the algebra $\mathscr{O}_{n}$ of germs of analytic functions $\left(\mathbb{C}^{n}, 0\right) \rightarrow \mathbb{C}$. We then set

$$
\mathbb{D}_{X}=\left\{D \in \mathbb{D}, D\left(I_{X}\right) \subset I_{X}\right\},
$$

where $I_{X} \subset \mathscr{O}_{n}$ is the ideal of functions vanishing on $X$. This is a subalgebra of $\mathbb{D}$. It will be called the tangent algebra of $X$. In case $X$ is a nonreduced germ, simple examples show that the tangent algebra of $X$ and of its reduction $X_{\text {red }}$ may coincide. This limits our interest to the reduced case. In this context, two main problems arise:

- Characterize all Lie subalgebras $A \subset \mathbb{D}$ that are of the form $A=\mathbb{D}_{X}$ for a suitable $X$.

Received by the editors April 16, 1991 and, in revised form, August 28, 1991.

1991 Mathematics Subject Classification. Primary 13B10, 14B05, 17B65, 32B10, 57R25, 58A30.

This work was done during a visit of the second author at the University of Innsbruck. He thanks the members of the Mathematics Department for their hospitality. 
- Find out to what extent the abstract Lie algebra $\mathbb{D}_{X}$ determines the variety $X$.

\section{TANGent Algebras Were CHARACTERIZED By LIE ALgEBRA PROPERTIES}

In order to discuss the first problem let us fix some notation. A subalgebra $A$ of a Lie algebra $B$ will be called balanced (in $B$ ) if $A$ contains no ideal $\neq 0$ of $B$ but an element $a \neq 0$ such that

$$
[a, B] \subset A \text { and } \quad[[a, B], B] \subset A .
$$

A visible subalgebra of $B$ is a subalgebra $A$ that admits a chain of subalgebras

$$
A=A_{m} \subset A_{m-1} \subset \cdots \subset A_{0}=B
$$

such that $A_{k}$ is maximal balanced in $A_{k-1}$ for $k=1, \ldots, m$. In case $m=1$, i.e., if $A$ is a maximal balanced subalgebra of $B, A$ is called maximal visible. Note that these notions are of a purely Lie algebra theoretic nature.

For a finite family $\mathbf{X}=\left\{X_{1}, \ldots, X_{p}\right\}$ of germs $X_{i} \subset\left(\mathbb{C}^{n}, 0\right)$ let $\mathbb{D}_{\mathbf{X}}=\bigcap_{i} \mathbb{D}_{X_{i}}$ be the Lie algebra of vector fields tangent to all $X_{i}$ (the $X_{i}$ may be contained in each other). Our first result may be considered as a variation of the classical Frobenius Theorem in the singular case (see e.g., [N, 2.11]).

Theorem 1. Let $A \subset \mathbb{D}$ be a subalgebra.

(a) There is a set of germs $\mathbf{X}$ as above such that $A=\mathbb{D}_{\mathbf{X}}$ if and only if $A$ is a visible subalgebra of $\mathbb{D}$.

(b) There is a smooth germ $X \subset\left(\mathbb{C}^{n}, 0\right)$ different from $\varnothing$ and $\left(\mathbb{C}^{n}, 0\right)$ such that $A=\mathbb{D}_{X}$ if and only if $A$ is a maximal visible subalgebra of $\mathbb{D}$.

(c) There is an irreducible germ $X \subset\left(\mathbb{C}^{n}, 0\right)$ with an isolated singularity at 0 such that $A=\mathbb{D}_{X}$ if and only if $A$ is a maximal visible subalgebra of the algebra $\mathbb{D}_{0}$ of vector fields vanishing at 0 .

(d) There is an analytic germ $X \subset\left(\mathbb{C}^{n}, 0\right)$ such that $A=\mathbb{D}_{X}$ if and only if $A$ is geometric in $\mathbb{D}$, i.e. by definition, $A$ is visible in every subalgebra $B$ of $\mathbb{D}$ containing $A$.

Comments. (i) It is easy to see that the family $\mathbf{X}$ of germs $X_{i}$ is not unique. For example, if $\mathbf{X}$ is the set of irreducible components $X_{i}$ of some germ $X=\bigcup X_{i}$ one has $\mathbb{D}_{X}=\mathbb{D}_{\mathbf{X}}$. Moreover, $\mathbb{D}_{X}=\mathbb{D}_{X}$, Sing $X$ where Sing $X$ denotes the singular subspace of $X$. But in case $\mathbf{X}$ is an irredundant set of irreducible germs, i.e., deleting any germ from $\mathbf{X}$ alters $\mathbb{D}_{\mathbf{X}}$, the family $\mathbf{X}$ is uniquely determined by $\mathbb{D}_{\mathbf{X}}$. In particular, the variety $X$ of a maximal geometric subalgebra as in (b) and (c) is unique.

(ii) There is a relative version of Theorem 1 where $\mathbb{D}$ is replaced by $\mathbb{D}_{\mathbf{Z}}$ for some set of germs $\mathbf{Z}$ and where all varieties $\mathbf{X}$ associated to visible subalgebras of $\mathbb{D}_{\mathbf{Z}}$ are determined. Namely, a subalgebra $A$ of $\mathbb{D}_{\mathbf{Z}}$ is visible if and only if there is a set of irreducible germs $\mathbf{X}$ with $X_{i} \not \subset Z_{j}$ for all $i, j$ such that $A=\mathbb{D}_{\mathbf{Z}, \mathbf{X}}$. Theorem 1 represents the cases $\mathbf{Z}=\varnothing$, resp. $\mathbf{Z}=\{0\}$.

\section{SiNGULARITIES ARE DETERMINED BY THEIR TANGENT ALGEBRA}

We now turn to the second problem, the characterization of the isomorphism type of germs via their Lie algebra. If $X, Y \subset\left(\mathbb{C}^{n}, 0\right)$ are isomorphic then the associated Lie algebras $\mathbb{D}_{X}$ and $\mathbb{D}_{Y}$ are isomorphic. In fact, every isomorphism $X \rightarrow Y$ can be extended to an automorphism $\phi$ of $\left(\mathbb{C}^{n}, 0\right)$ with algebra 
automorphism $\phi^{*}: \mathscr{O}_{n} \rightarrow \mathscr{O}_{n}$. Then $\Phi(D):=\phi^{*} \circ D \circ\left(\phi^{*}\right)^{-1}$ defines an automorphism $\Phi$ of $\mathbb{D}$ with $\Phi\left(\mathbb{D}_{Y}\right)=\mathbb{D}_{X}$. By abuse of notation we write again $\Phi=\phi^{*}$. This map is continuous if $\mathbb{D}$ is provided with the topology induced from the coefficientwise topology on $\mathscr{O}_{n}$. Conversely we have

Theorem 2. Let $X$ and $Y$ be germs of analytic varieties in $\left(\mathbb{C}^{n}, 0\right)$ different from $\varnothing$. Assume that $n \geq 3$. For every isomorphism $\Phi: \mathbb{D}_{Y} \rightarrow \mathbb{D}_{X}$ of topological Lie algebras there is a unique automorphism $\phi$ of $\left(\mathbb{C}^{n}, 0\right)$ sending $X$ onto $Y$ and such that $\Phi=\phi^{*}$.

Thus the analytic isomorphism type of $X$ is entirely given by the abstract topological Lie algebra $\mathbb{D}_{X}$. Omori [O] proved this in the special case of weighted homogeneous varieties.

We indicate some ideas appearing in the proofs of Theorems 1 and 2.

\section{Proof of Theorem 1}

In order to study visible subalgebras of $\mathbb{D}$ we associate to any $A \subset \mathbb{D}$ the germ $X(A)$ in $\left(\mathbb{C}^{n}, 0\right)$ defined by the ideal $\sqrt{I(A)}$ of $\mathscr{O}_{n}$ where

$$
I(A)=\left\{g \in \mathscr{O}_{n}, g \cdot \mathbb{D} \subset A\right\} .
$$

Here the $\mathscr{O}_{n}$-module structure of $\mathbb{D}$ is used. The germ $X(A)$ will be called the integral variety of $X$. Note that every germ $X \subset\left(\mathbb{C}^{n}, 0\right)$ different from $\varnothing$ and $\left(\mathbb{C}^{n}, 0\right)$ can be recovered from $\mathbb{D}_{X}$ as $X=X\left(\mathbb{D}_{X}\right)$ : The inclusion $X\left(\mathbb{D}_{X}\right) \subset X$ is obvious from the definition. For the converse, assume that some $g \in I\left(\mathbb{D}_{X}\right)$ does not belong to $I_{X}$. Consider the vector fields $g \partial_{x_{1}}, \ldots, g \partial_{x_{n}}$. In every point outside the zero set of $g$ in $X$ they are linearly independent. As they are tangent to $X$ by definition of $I\left(\mathbb{D}_{X}\right)$ a Theorem of Rossi [R, Theorem 3.2] implies that the germ of $X$ taken in such a point is isomorphic to $\left(\mathbb{C}^{n}, 0\right)$. But these points are dense in $X$ and we get a contradiction.

Let us now consider assertion (b) of Theorem 1 . The proof that $\mathbb{D}_{X}$ is a balanced subalgebra of $\mathbb{D}$ is a bit involved and will be left out. Concerning maximality, assume that $\mathbb{D}_{X}$ is contained in a balanced subalgebra $A \subset \mathbb{D}$. Then in fact $\mathbb{D}_{X} \subset A \subset \mathbb{D}_{X(A)}$. One shows that $A$ balanced implies $X(A) \neq \varnothing$ and $\left(\mathbb{C}^{n}, 0\right)$. Moreover $X(A)=X\left(\mathbb{D}_{X(A)}\right) \subset X\left(\mathbb{D}_{X}\right)=X$. Now if $X$ is smooth one deduces from $\mathbb{D}_{X} \subset \mathbb{D}_{X(A)}$ that $X(A)=X$. This shows $\mathbb{D}_{X}=A$ and proves necessity in (b).

For sufficiency, start with a maximal visible subalgebra $A \subset \mathbb{D}$. Similarly as above $A \subset \mathbb{D}_{X(A)}$ with $X(A) \neq \varnothing$ and $\left(\mathbb{C}^{n}, 0\right)$. As $\mathbb{D}_{X(A)}$ is balanced, maximality of $A$ gives $A=\mathbb{D}_{X(A)}$. Write $X=X(A)$. If Sing $X \neq \varnothing$ then $\mathbb{D}_{\text {Sing } X}$ is balanced. Again by maximality, the inclusion $\mathbb{D}_{X} \subset \mathbb{D}_{\text {Sing } X}$ is actually an equality. This implies $X=\operatorname{Sing} X$, which is impossible. Therefore $X$ is smooth.

Part (a) of Theorem 1 is proved by induction. Here one proves and uses at once the relative version of the theorem mentioned earlier. To illustrate, let $\mathbf{X}=\{X\}$ consist of one singular germ $X$. Choose $k \in \mathbb{N}$ maximal with $Z=\operatorname{Sing}^{k} X:=\operatorname{Sing}\left(\cdots(\operatorname{Sing}(X)) \neq \varnothing\right.$. The inclusion $\mathbb{D}_{X} \subset \mathbb{D}$ is split into $\mathbb{D}_{X}=\mathbb{D}_{Z, X} \subset \mathbb{D}_{Z}$ and $\mathbb{D}_{Z} \subset \mathbb{D}$. The first is visible by induction and the second is maximal visible by (b) since $Z$ is smooth. 
Conversely, if $A$ is a visible subalgebra of $\mathbb{D}$ use induction on the length of the chain and the relative version of part (b) to find $\mathbf{X}$.

\section{Proof of Theorem 2}

We conclude with some remarks on the proof of Theorem 2. For $f \in \mathscr{O}_{n}$ consider the $\mathbb{C}$-linear map $\lambda_{f}: \mathbb{D}_{X} \rightarrow \mathbb{D}_{X}$ defined by

$$
\lambda_{f}(D)=\Phi\left(f \cdot \Phi^{-1}(D)\right) .
$$

If $\Phi: \mathbb{D}_{Y} \rightarrow \mathbb{D}_{X}$ is induced from an automorphism $\phi$ of $\left(\mathbb{C}^{n}, 0\right)$, say $\Phi=\phi^{*}$, one checks by computation that the equality $\lambda_{f}(D)=\phi^{*}(f) \cdot D$ holds for all $D$ in $\mathbb{D}_{X}$. If $\Phi$ is an arbitrary continuous Lie algebra isomorphism, we are led to establish the same equality in order to recover a map $\phi$ that could be an appropriate candidate to induce $\Phi$ and to define an isomorphism between $X$ and $Y$.

Thus the first thing to do is to check whether any vector field $D$ is mapped by $\lambda_{f}$ into the $\mathscr{O}_{n}$-module $(D)$ generated by $D$. This can be seen for all $D$ of a certain dense subset $U$ of $I_{X} \cdot \mathbb{D}$ by writing $(D)$ as an intersection of subalgebras of $\mathbb{D}_{X}$ of form $\mathbb{D}_{X, Z}$. This is the key step in the proof and it is here that we need the assumption $n \geq 3$. Once this is accomplished, the relative version of Theorem 1 and the fact that $\Phi$ is a Lie algebra isomorphism guarantee that $\lambda_{f}$ maps $\mathbb{D}_{X, Z}$ into $\mathbb{D}_{X, Z}$. Hence the module $(D)$ is mapped into itself. This implies

$$
\lambda_{f}(D)=\phi^{*}(f, D) \cdot D
$$

with suitable factor $\phi^{*}(f, D) \in \mathscr{O}_{n}$. Then the continuity of $\Phi$ is used to show that $\phi^{*}(f, D)$ is actually independent of $D$, say $\phi^{*}(f, D)=\phi^{*}(f)$. Therefore, again by continuity,

$$
\lambda_{f}(D)=\phi^{*}(f) \cdot D
$$

will hold for all $D \in I_{X} \cdot \mathbb{D}$. Finally we deduce from this equality that the map $\phi$ thus obtained is an automorphism of $\left(\mathbb{C}^{n}, 0\right)$ mapping $X$ to $Y$ and inducing $\Phi$.

\section{REFERENCES}

[HM1] H. Hauser and G. Müller, Analytic varieties and Lie algebras of vector fields. Part I: The Gröbner correspondence, preprint 1991. To be published.

[HM2] __, Analytic varieties and Lie algebras of vector fields. Part II: Singularities are determined by their tangent algebra (to appear).

[N] R. Narasimhan, Analysis on real and complex manifolds, North Holland, Amsterdam, 1968.

[O] H. Omori, A method of classifying expansive singularities, J. Differential Geom. 15 (1980), 493-512.

[R] H. Rossi, Vector fields on analytic spaces, Ann. of Math. (2) 78 (1963), 455-467.

MATHEMATISCHES INSTITUT DER UNIVERSITÄt INNSBRUCK, A-6020 INNSBRUCK, AUSTRIA

Fachbereich Mathematik der Universität Mainz, D-6500 Mainz, Germany 